\title{
FLARE STARS AS AGE INDICATORS IN OPEN CLUSTERS
}

\author{
L.PIGATTO \\ Osservatorio Astronomico \\ Vicolo dell'Osservatorio, 5 \\ 35122 Padova \\ Italy
}

ABSTRACT. A new set of isochrones derived from overshooting evolutionary models, allows one to give a homogeneous age determination for open clusters with solar chemical composition and in a range of age $410^{7} \div 1.510^{9} \mathrm{yr}$. In this context is derived an empirical new age calibration - absolute magnitude of the supposed brightest flare star in a cluster versus log age - . The evolutionary implication in finding or not finding flare stars in candidate open clusters is also examined.

\section{Introduction}

The main tools in clusters age determination are the isochrones, the same timelines, computed from the evolutionary models of the same chemical composition. In these last years the evolutionary models have come to reach an higher accuracy because of new and up-to-date physical inputs: but some controversies are still open among modelers about the best evolutionary models capable of explaining some features in the clusters HR diagram not understandable in the classical evolutionary scheme. We think that the best models are those which are able to explain the greatest number of evolutionary problems as a whole. In this context the overshooting evolutionary models and the derived isochrones (Bertelli et al. 1986) were able to account for several questions about the HR diagram characteristics in open clusters (Mazzei and Pigatto, 1988, 1989 and references therein).

\section{Age determination in open clusters}

Let us concentrate on the problems of the young and intermediate-age open clusters. Mazzei and Pigatto (1988) put into evidence the necessity of a correct method in the clusters age determination by using not only the pure best-fit technique, but also comparing the observed and theoretical luminosity functions, finally deriving from the isochrones synthetic clusters which are able to mimic the morphology of the observed HR diagrams. The age of the Pleiades derived by this method is of $1.510^{8} \mathrm{yr}$ (Mazzei and Pigatto,1989). The uncertainty in the Pleiades age determination is of about $50 \%$; but a better determination of about $10 \%$ of uncertainty can be derived for intermediate-age open clusters (Mazzei and Pigatto, 
1988) where the presence of the red giants clump provides an additional constraint in the age calibration. In the same way the age of the open cluster NGC 7092 is here derived by using the overshooting isochrones for solar chemical composition. The choice of this cluster will be discussed in the next section. Figure la shows the colour-magnitude diagram of NGC 7092 in which, for the assigned age, the $610^{8}$ $\mathrm{yr}$ old isochrone is overimposed.
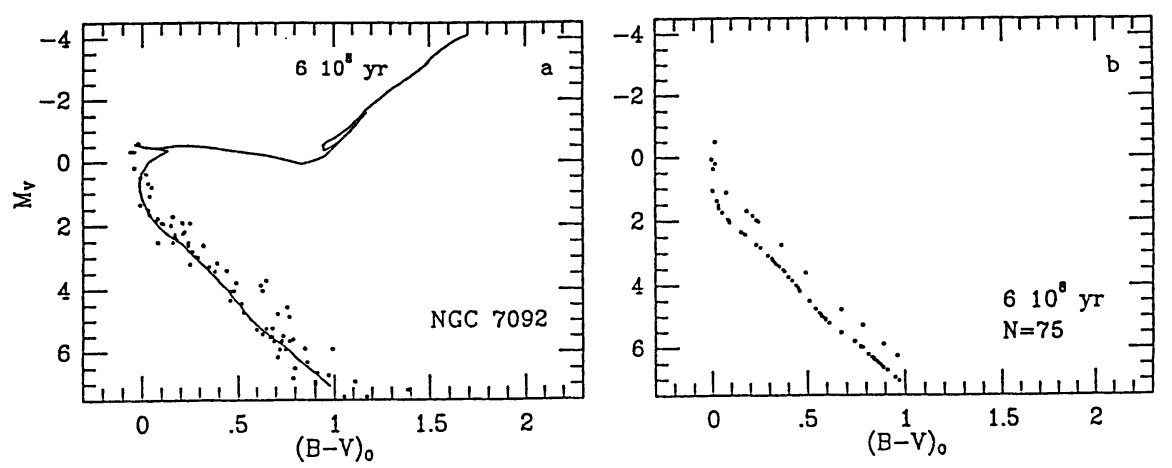

Figure 1. The colour-magnitude diagram of NGC 7092 (a) and overimposed the overshooting isochrone of $610^{8} \mathrm{yr}$ with solar chemical composition and a synthetic cluster (b) from the same isochrone for $\mathrm{N}=75$ objects.

To support the reliability of this age determination, a synthetic cluster chosen for the best morphological resemblance from a group of samples computed from the 5,6 and $710^{8} \mathrm{yr}$ old isochrones respectively is shown in fig. $1 \mathrm{~b}$.

\section{Flare stars as age indicators}

Usually ages in clusters are derived by the analysis of the brilliant Main-Sequence (MS) portion just above and around the turnoff region, but it is also interesting, from the evolutionary point of view, to examine the fainter MS portion, i.e. the turnon region, where the stars are just arriving in the MS after the contraction phase. The flaring activity is generally associated with this evolutionary phase, and the relation with the clusters age is well demonstrated by many observational facts such as the earliest spectral type and/or the brightest magnitude of the flare stars in a cluster. Parsamian $(1976,1985)$ first put into evidence the dependence on the age of the absolute magnitude $\mathrm{M}_{\mathrm{f}}$ of the brightest flare star in a cluster or aggregate and the possible monotonous behaviour of this relation. Figure 2 shows the relation between the brightest flare star (Haro,1968 and references therein) versus $\log t$, in three well known clusters as NGC 2264, the Pleiades and the Praesepe (full squares). Ages are derived from the turnoff overshooting isochrones. Because of the new age and because of a distance modulus of $5.88 \mathrm{mag}$ by Pérez et al.(1988), NGC 2264 is correctly located in the linear correlation $M_{f}, \log t$. The reason for this empirical and rather uncertain calibration, is based on a first attempt at listing open clusters in which flare stars could be looked for. In this calibration one fact is evident: in the range of age $10^{8} \div 10^{9} \mathrm{yr}$, from the Pleiades 
to the Praesepe, no open clusters with known flare stars are present.

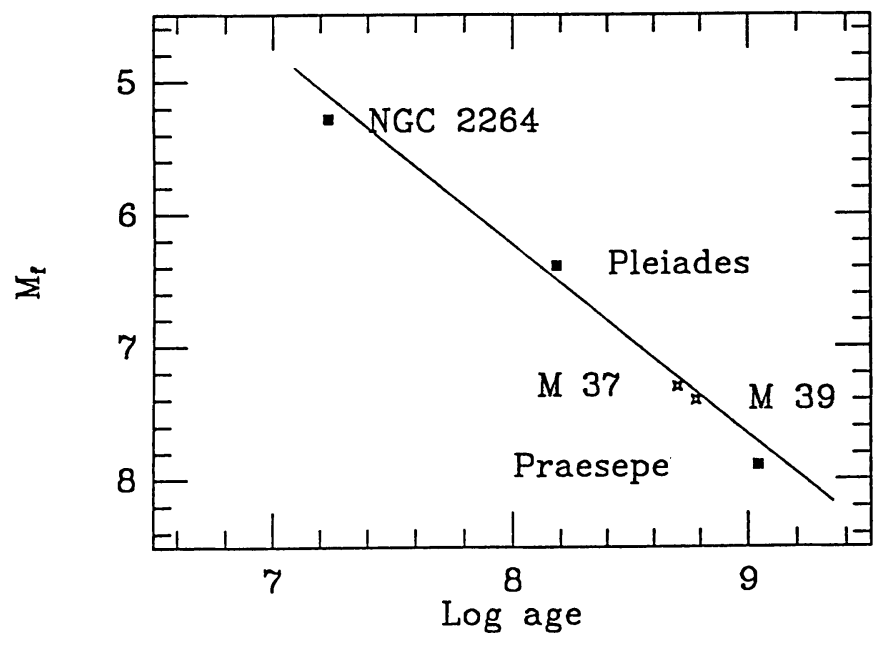

Figure 2. Absolute magnitude of the brightest flare star versus log age. The ages are derived from the overshooting isochrones for solar chemical composition.

The first reason can refer simply to the fact that a detectability of a flare is connected with its amplitude and with the cluster distance. Another reason can be due to the fact that in a cluster not very young and with very few stars, the probability of finding flare stars is very low. In fact, the flare activity is going to reduce and then disappears in the oldest clusters where even the low mass stars have already arrived on the MS. From the calibration the supposed apparent magnitude of the brightest flare stars for NGC 2099 and NGC 7092 has been derived taking into account the reddening and the distance modulus (photometric data are from West, 1967 and Platais, 1984 respectively). The not very faint value of this magnitude (14.5 mag) for NGC 7092 suggests that this cluster can be a good candidate for the search of flare stars. NGC 7092 (M 39) was believed peculiar by Mc Namara and Sanders (1977) because of the lack of faint stars in its HR diagram. A more recent research by Platais (1984) has shown stars in the low MS down to the $15^{\text {th }}$ mag. If the flare activity is an obligatory step in the evolutionary path of the stars in a cluster, flare stars can be expected in NGC 7092, accounted for the dependence on the number of the cluster stars and on the observation time. On the other hand the lack in finding flare stars in the cluster area could give interesting information on the mass segregation and on the cluster relaxation time.

\section{Conclusions}

To discover flare stars in open clusters can signify answering many still open questions such as: 1) turnon identification and age determination from pre-mainsequence models, 2) rotation, binarity and/or multiplicity and their influence on the flare stars position in the HR diagram, 3) eventual difference between the turnoff 
and turnon age in a cluster as indicator of a possible bimodal star formation, 4) mass segregation and relaxation time in open clusters.

\section{References}

Bertelli,G., Bressan,A., Chiosi,C., Nasi, E., Pigatto, L.:1986, preliminary draft Crawford,D.L., Barnes, J.V.:1969, Astron. J. 74, 818

Haro, G.: 1968, in Stars and Stellar Systems, eds. B.M. Middlehurst, L.H. Aller, Chicago, 7, p. 141

Johnson, H.L., Mitchell, R.I.:1958, Astrophys. J. 128, 31

Mc.Namara, B.J., Sanders, W.L.: 1977, Astron. Astrophys. Suppl. 30, 45

Mazzei, P., Pigatto, L.:1988, Astron. Astrophys. 193, 148

Mazzei, P., Pigatto, L.:1989, Astron. Astrophys. 213, L1

Parsamian, E.S.: 1976, Astrofisika 12, 235

Parsamian, E.S.: 1985, Rev. Mex. Astr. Astr. 10, Spec. Issue, p. 221

Pérez, M.R., The, P.S., Westerlund, B.E.: 1987, PASP 99, 1050

Platais, I.K.: 1984, Sov. Astron. Lett. 10, 84

West, R.F.: 1967, Astrophys. J. Suppl. 14, 359

LANG: What are the uncertainties in your age determinations? Why aren't there any young stars above or below the main sequence in your HRdiagram?

PIGATTO: The uncertainties are 50 per cent. Only the brightest stars were plotted in my HR-diagrams; they show no stars off the main sequence, so such behavior only applies to the low luminosity stars. 